\title{
Erratum to: The Effect of Conditional Discrimination Reversals with SMTS and DMTS on Reorganization of Equivalence Classes
}

\author{
Giovan W. Ribeiro $^{1}$ - Marcelo V. Silveira ${ }^{1} \cdot$ Harry A. Mackay $^{2} \cdot$ Julio C. de Rose $^{1}$
}

Published online: 11 November 2016

(C) Association for Behavior Analysis International 2016

Erratum to: Psychol Rec

DOI: 10.1007/s40732-016-0194-Z

Table 3 , as originally published, is incorrect. The label DMTS was applied for both groups. The correct version of Table 3 is represented below.

The online version of the original article can be found at http://dx.doi. org/10.1007/s40732-016-0194-z.

\footnotetext{
Marcelo V. Silveira marcelopsi06@gmail.com

$\triangle$ Julio C. de Rose juliocderose@yahoo.com.br

1 Departamento de Psicologia, Universidade Federal de São Carlos, Via Washington Luis Km 235, São Carlos, SP 13565-905, Brazil

2 Eunice Kennedy Shriver Center-New England Center for Children, 33 Turnpike RD, Southborough 01772, MA, USA
} 
Table 3 Participants' performances on training and testing blocks of the second phase of the experiment

\begin{tabular}{|c|c|c|c|c|c|c|c|c|c|c|c|}
\hline \multirow[b]{3}{*}{ Groups } & \multirow[b]{3}{*}{ Participants } & \multicolumn{6}{|c|}{ AD Reversal } & \multicolumn{3}{|c|}{ Cumulative Baseline with AD Reversals } & \multirow{3}{*}{$\begin{array}{l}\text { Reorganization Test } \\
\text { Session } 1 \\
\text { B1. } 1\end{array}$} \\
\hline & & \multicolumn{2}{|c|}{ Session 1} & \multicolumn{2}{|c|}{ Session 2} & \multicolumn{2}{|c|}{ Session 3} & \multirow{2}{*}{$\begin{array}{l}\text { Session } 1 \\
\text { Bl. } 1\end{array}$} & \multirow{2}{*}{$\begin{array}{l}\text { Session } 2 \\
\text { Bl. } 1\end{array}$} & \multirow{2}{*}{$\begin{array}{l}\text { Session } 3 \\
\text { B1. } 1\end{array}$} & \\
\hline & & B1. 1 & B1. 2 & Bl. 1 & B1. 2 & B1. 1 & B1. 2 & & & & \\
\hline \multirow[t]{8}{*}{ SMTS } & $\mathrm{P} 1$ & 67 & 100 & - & - & - & - & 86 & 97 & 97 & 75 \\
\hline & $\mathrm{P} 2$ & 83 & 92 & 100 & 100 & - & - & 92 & 97 & 97 & 100 \\
\hline & P3 & 92 & 100 & - & - & - & - & 86 & 100 & - & 100 \\
\hline & $\mathrm{P} 4$ & 83 & 100 & - & - & - & - & 92 & 100 & - & 100 \\
\hline & P5 & 83 & 100 & & - & - & - & 86 & 92 & 94 & 33 \\
\hline & P6 & 92 & 100 & - & - & - & - & 92 & 100 & - & 100 \\
\hline & P7 & 67 & 100 & - & - & - & - & 50 & 97 & 97 & 100 \\
\hline & P8 & 92 & 100 & - & - & - & - & 94 & 100 & - & 100 \\
\hline \multirow[t]{8}{*}{ DMTS } & P9 & 75 & 83 & 92 & 92 & 100 & 100 & 75 & 92 & 92 & 91 \\
\hline & $\mathrm{P} 10$ & 75 & 100 & - & - & - & - & 86 & 97 & 92 & 33 \\
\hline & P11 & 58 & 100 & - & - & - & - & 86 & 92 & 97 & 94 \\
\hline & P12 & 83 & 92 & 100 & 92 & 100 & 92 & 89 & 97 & 97 & 88 \\
\hline & P13 & 75 & 100 & - & - & - & - & 92 & 94 & 100 & 91 \\
\hline & P14 & 83 & 100 & - & - & - & - & 92 & 100 & - & 97 \\
\hline & P15 & 0 & 0 & 0 & 92 & 92 & 92 & 86 & 92 & 92 & 83 \\
\hline & P16 & 92 & 100 & - & - & - & - & 92 & 97 & 100 & 100 \\
\hline
\end{tabular}

